\title{
EL ALIVIO DE LA POBREZA EN MÉXICO, UN ASUNTO INCONCLUSO: DE LA BENEFICENCIA A LA INVERSIÓN EN CAPITAL HUMANO
}

\author{
Oscar Alfonso Martínez Martínez ${ }^{1}$ \\ Claudia Campillo Toledano ${ }^{2}$
}

\section{Resumen}

La pobreza en México es una problemática histórica la cual ha sido tratada de diversas maneras, desde la caridad por las instituciones eclesiales hasta los programas de inversión en capital humano. Cada estrategia ha buscado en esencia resolver el problema de la pobreza, pero en la práctica han tenido poco impacto, debido a que no responden a las necesidades reales de un país tan diverso como México. Por tanto en el presente artículo se discuten las estrategias de combate a la pobreza que se han aplicado al mismo tiempo se muestran las disyuntivas que las mismas han presentado y que finalmente han repercutido en el bienestar de las personas que viven bajo las condiciones más precarias de pobreza y marginación.

Descriptores: Pobreza, política social, inversión en capital humano.

\section{Abstract}

Poverty in Mexico is a historic problem which has been treated in various ways, from charity institutions for church programs up capital investment smoke. Each strategy has sought to fundamentally solve the problem of poverty, but in practice have had little impact because they do not respond to

\footnotetext{
${ }^{1}$ Profesor-Investigador del Departamento de Ciencias Sociales y Políticas de la Universidad Iberoamericana, Ciudad de México.

2 Profesora-Investigadora de la Facultad de Trabajo Social y Desarrollo Humano de la Universidad Autónoma de Nuevo León.
} 
the real needs of a country as diverse as Mexico. Therefore in this article we discuss the strategies to combat poverty that have been implemented at the same time shows the choices that they have presented and ultimately have an impact on the well-being of people living under the most precarious poverty and marginalization.

Key words: Poverty, social policy, investment in human capital.

\section{Introducción}

Los programas de combate a la pobreza que se han aplicado en México han pasado por una serie de modificaciones a lo largo de la historia no solo respecto a la dimensión operativa sino a la forma de resolver dicha problemática. En los orígenes del tratamiento a la pobreza se consideraban más bien acciones de caridad hacia los desprotegidos pero con el paso del tiempo se fue modificando hasta llegar a los programas de inversión en capital humano, donde se busca romper con el círculo intergeneracional de la pobreza por medio de la aplicación de recursos al interior de las familias para que se utilicen en gastos relacionados con alimentación y salud. Siendo que el concepto de pobreza así como la forma de combatirla ha evolucionado; ese proceso de cambio paulatino hace cuestionar si ilos programas de alivio a la pobreza en México han respondido a las necesidades sociales que se vivían en los distintos momentos históricos en los que se aplicaron? Para responder a dicha incógnita es necesario hacer un análisis sobre las diversas etapas por las que han pasado la política social de combate a la pobreza y con ello precisar si estás han ayudado o no al mejoramiento social.

Para contestar la interrogante planteada, el presente documento se ha dividido en tres secciones. En la primera se hace un análisis sobre las principales acciones emprendidas por instituciones como la Iglesia y posteriormente por el gobierno, para mejorar las condiciones de vida de las personas que vivían en pobreza y marginación, las cuales tenían un sesgo racial. En la segunda se discuten los primeros programas de combate a la pobreza, los cuales se caracterizaban por su corrección a lo largo del tiempo a base de prueba y error, en dicho periodo también existió un lapso de tiempo que lo hemos denominado como el periodo muerto de las políticas de combate a la pobreza por el desmantelamiento de los programas existentes debido a las condiciones económicas que se vivieron en ese momento. En la tercera sección se analiza cada una de las partes de este programa 
gubernamental y la forma en como éste sustenta que el desarrollo de las capacidades permitirán lograr el combate a la pobreza.

Desde el discurso oficial se ha reconocido que este programa está basado en la premisa de que "una de las razones fundamentales de la reproducción intergeneracional de la pobreza es la falta de inversión en capital humano en los ámbitos de educación, salud y nutrición" (Villatoro, 2004:9). Sin embargo a pesar de todos los puntos a favor del programa como de la teoría misma que lo sustenta es importante discutir la problemática que implica una inversión en los seres humanos, debido a que para su éxito es necesario el tener fuentes de empleo o autoempleo suficiente para introducir al mercado a las personas en quienes se invirtió, lo cual ha resultado complejo debido a la poca o nula vinculación entre las políticas de desarrollo social y las políticas laborales. Finalmente, se presentan las conclusiones donde se discuten las posibles respuestas a la pregunta planteada en este trabajo.

\section{De la beneficencia a los Gobiernos Posrevolucionarios}

En el presente apartado se analizan los principales aportes a la política social de combate a la pobreza aplicados en México desde la colonia hasta los primeros gobiernos posteriores a la revolución de 1910. A lo largo de este periodo se observa como las estrategias para contrarrestar este problema sufre modificaciones sobre todo en el periodo posrevolucionario, sin embargo los resultados que se pretendían lograr no se pudieron alcanzar, trayendo como consecuencia que se hiciera más grande la brecha de pobreza y marginación entre las áreas rurales y las urbanas.

Las políticas de alivio a la pobreza en México tienen sus orígenes a partir de la colonia, a través de la beneficencia o caridad, ya que previo a ese momento no existían programas específicos con esta finalidad. Es también por ello que el concepto de pobre como tal aun no es utilizado, en su lugar se utilizaba el de miserable, el cual representaba a todas aquellas personas que vivían bajo condiciones de precariedad. Las estrategias para mejorar las condiciones sociales de estas personas eran por tanto limitadas ya que consistían en la creación de hospitales, hospicios y posteriormente asilos así como colegios. Estos fueron proporcionados en su gran mayoría por la Iglesia Católica y en menor número por el Estado así como por algunos particulares por medio de cofradías. 
Los hospitales, hospicios y asilos, que en esa época albergaban a las personas consideradas como miserables poseían una fuerte carga de tradición proveniente de las hospederías medievales en las que recibían a los pobres, enfermos y necesitados, y se les proporcionaban alimentos, cuidados médicos, y asistencia religiosa (Villarespe, 2001). Esta última, debido a que la Iglesia Católica tenía a su cargo tal misión en las nuevas tierras conquistadas. A pesar de que las estrategias realizadas en esencia eran nobles éstas tenían varias limitantes ya que para ingresar a cualquiera de estos lugares existía una marcada diferenciación de acuerdo a la raza, pues las castas estaban protegidas por legislaciones distintas y los más desprotegidos eran los nativos de dicho país.

En 1763 inicia un proceso de mejoramiento (al menos en esencia) para quienes habían sido desprotegidos por las distintas legislaciones de acuerdo a la raza, buscando de esta forma que todas las personas consideradas como miserables fueran cubiertas por los mismos beneficios y en igualdad de condiciones sin importar a cual pertenecían (castas). Para lograrlo se fundó el Hospicio de los Pobres con el propósito de que fuera un asilo para ancianos y mendigos, sin embargo, estos lugares resultaron iguales a los asilos-talleres o "work-houses" de Inglaterra, pues en ellos se establecían talleres donde existía una sobreexplotación del trabajo bajo el pretexto de enseñarles un oficio. Estos lugares se volvieron más bien como pequeños guetos para los pobres donde se les daban techo y comida a cambio de trabajo, el cual era realizado en condiciones precarias y en horarios excesivamente largos, de ahí que la igualdad lograda en realidad fue en términos de otorgarles trabajo excesivo y precarias condiciones. Por tanto la estrategia en términos de un mejoramiento social en el largo plazo fue poco exitoso, además que las personas dejaron de asistir a dichos lugares por las malas condiciones que ahí se vivían.

En 1775 ante el poco éxito logrado por los asilos para ancianos y mendigos, se fundó el Monte de Piedad, uno de sus principales objetivos era otorgar préstamos a los pobres y dedicar ganancias a obras de caridad (Villarespe, 2001). Aunque esta opción en apariencia era buena se enfrentaba a un gran problema, debido a que las personas más pobres e incluso los vagabundos no contaban con bienes que pudieran empeñar para obtener un ingreso que les ayudara a mejorar su situación, por tanto resultó paradójico pues la institución se enfocó solo en los pobres que poseían bienes que estuvieran en buen estado y que pudieran dejar en garantía. Un punto a favor de dicha estrategia fue que los recursos obtenidos se aplicaban a obras de 
beneficencia, pero estás se realizaron principalmente en la capital del país descuidándose las entidades federativas.

En los diversos intentos para mejorar a las instituciones que ayudaban a los individuos más pobres se inauguró en 1879 el asilo Francisco Díaz de León el cual en su inicio fue exclusivamente para mendigos (Villarespe, 2001), donde se buscó distinguir claramente a los mendigos sanos de los ociosos. Esto pudo haber sido un reflejo de la discusión habida en Inglaterra por la Ley de los Pobres sobre los que eran dignos y no dignos para recibir ayuda: los primeros (dignos) eran aquellos que querían trabajar pero no podían y los indignos los que no querían trabajar a pesar de poder hacerlo, y por tanto estos últimos no se les consideraba para recibir cualquier tipo de asistencia. Sin embargo el proceso de identificación era muy rudimentario pues no se tenía claro qué aspectos tendrían que definir a quien incluir o no, lo que podría excluir a personas que lo necesitaban e incluir a aquellas que no lo requerían.

Es de esta forma que desde la época colonial hasta tiempo después de la independencia en el 1810, las acciones de alivio a la pobreza no tuvieron el impacto que se hubiera esperado debido a que las estrategias emprendidas no eran las más acordes a las condiciones sociales que se vivían. Lo anterior se debe a varios factores como la falta de infraestructura en diversos lugares del país pues los hospitales e instituciones que se crearon con este fin por lo regular se establecieron en las grandes ciudades sobre todo en el Distrito Federal, cuando en ese momento México era eminentemente rural y los más empobrecidos estaban en las áreas rurales. Esta situación trajo un mayor empobrecimiento y desigualdad social entre estos dos contextos, que aun se vienen arrastrando hasta nuestros días.

En el periodo de las leyes de reforma con Benito Juárez, la noción de la caridad se transforma de virtud privada otorgada principalmente por el clero católico en un servicio público proporcionado por la administración civil. Estos planteamientos produjeron modificaciones al patrón ideológico, eminentemente religioso el cual se sustituyó por una perspectiva laica. Estos cambios ideológicos buscaban desde el discurso, sentar las bases para instruir una beneficencia moderna y accesible a los grupos necesitados del país, y cuyas estrategias principales consistían en: Introducir en las casas de beneficencia, la enseñanza de algunos oficios; autorizar la fundación de la lotería nacional para que el $15 \%$ de sus ingresos se aplicara a los 
orfanatorios; establecer un hospital de maternidad e infancia; la centralización de los servicios de socorro y su control por parte del Estado (Villarespe, 2001).

En el fondo estas estrategias tuvieron como finalidad restarle a la Iglesia su marco de acción, ya que hasta entonces era la encargada de realizar las pocas acciones en contra de la pobreza. Como menciona al respecto Pardo (2000:462) "el compromiso social del Estado de responsabilizarse por los desprotegidos no aparece como resultado de las reformas liberales, sino se explica más como una reacción frente a la Iglesia católica". Se logró de esta manera, una disminución en los ámbitos de acción de la Iglesia, al mismo tiempo que se dio su separación con el Estado, lo cual produjo beneficios de índole legal y administrativa pero condujo a una serie de problemas que empeoraron la situación de marginación en que se vivía. Debido a que se expropiaron las tierras del clero mediante la nacionalización de bienes Eclesiásticos, éstas no pudieron seguir siendo productivas ya que el gobierno carecía de los recursos suficientes para trabajarlas, lo que provocó que los campesinos que dependían de éstas para su sobrevivencia se enfrentaran a una realidad más adversa.

En el periodo de la revolución mexicana el país atravesó una fuerte inestabilidad económica y política, lo que trajo como resultado que los primeros gobiernos posrevolucionarios concedieran poca importancia al combate a la pobreza, centrando sus esfuerzos en el control militar, la estructuración del sistema político, los derechos de los trabajadores (salarios, prestaciones y condiciones mínimas de trabajo), el fomento económico y sobre todo en el reparto de las tierras (Ordóñez, 2002). Por tanto la pobreza acumulada desde años atrás sumada a la que fue originada por los estragos de la revolución tenía como resultado un escenario de alta marginación en todo el país. La atención a la pobreza no resultó prioritario para los primeros gobiernos posrevolucionarios, ocupando una posición secundaria en la agenda gubernamental debido principalmente a la debilidad organizativa y financiera que padecía el Estado.

Una vez terminada la revolución mexicana, las acciones emprendidas por los primeros gobiernos posrevolucionarios en cuanto al alivio de la pobreza fueron escasos, careciendo de una visión sobre la forma en como combatirla. De ahí que durante la presidencia de Venustiano Carranza (1917-1920), las acciones a favor de los pobres redundaron en dos hechos. Primero, se creó el 
Consejo de Salubridad General (con el fin de impulsar campañas contra el alcoholismo y la drogadicción) y el Departamento de Salubridad General (para combatir epidemias y enfermedades exóticas). En segundo lugar desapareció el antiguo Ministerio de Instrucción Pública y Bellas Artes del porfiriato por considerar que la educación pública no universitaria debía ser impartida por los ayuntamientos y el gobierno del Distrito Federal. Estas dos estrategias buscaban un mejoramiento en la salud y educación de la población, sin embargo el principal problema que enfrentaban las personas en condiciones de pobreza era el debilitamiento de su economía que se manifestaba en su bajo poder adquisitivo debido a los estragos del conflicto armado en el país. El que no pudieran adquirir los alimentos básicos necesarios, aunado a la constante escasez de los mismos, generaba que las familias enfrentaran altos grados de marginación y pobreza.

En el siguiente gobierno que fue el de Álvaro Obregón (1920-1924) las acciones se concentraron en dos objetivos: el ampliar la educación primaria, creándose la Secretaría de Educación Pública en 1921 con la misión de extender la educación primaria gratuita; el segundo fue promover la creación de unidades sanitarias no especializadas y la instrumentación de medidas preventivas así como garantizar una pensión a los empleados civiles y militares de la federación (Ordóñez, 2002). Ambas estrategias iban sentando las bases de una identidad de las acciones que el gobierno mexicano emprendía para el alivio a la pobreza, pero todo esto era aun poco en relación a las carencias que se vivía en el país, ya que el discurso de justicia social y universalista que promovía la revolución y que quedó plasmado en la constitución, se iba perdiendo debido a que las prioridades eran el control militar, la estructuración del sistema político y el fomento económico. De ahí que la postura utópica de garantías constitucionales que harían un México con menos desigualdades al mejorar la situación de las personas que por años habían vivido bajo pésimas condiciones no pudo materializarse.

Uno de los momentos más importantes en las estrategias de alivio a la pobreza dentro de los gobiernos posrevolucionarios se dio en la década de los '30, siendo presidente Lázaro Cárdenas. Este cambió la filosofía política, centrando su posición en que la solución de la marginación económica y social era cuestión de interés nacional, trajo como consecuencia cambios fundamentales en el concepto de beneficencia que se sustituyó por el de asistencia, implicando con ello que son obligaciones del Estado el promover y regular las acciones relativas a la salud y el proporcionar auxilio médico y 
social a la población. Es por esta razón que se empiezan a destinar recursos al bienestar social (aunque todavía no se habla de programas de combate a la pobreza). Las acciones emprendidas fueron:

- Establecer un asilo-hospital en el que se internaba a los mendigos y posteriormente sólo a los enfermos crónicos.

- Imponer sanciones penales a quienes ejercieran la mendicidad profesional. Se realizó una intensa propaganda con el lema: no dé limosna de trabajo.

- Crear un establecimiento que tuviera una granja anexa y pequeños talleres, para que los mendigos pudieran realizar algún trabajo (Villarespe, 2001).

El cambio más importante sin duda se da en 1934 al promulgarse una reforma al artículo $3^{\circ}$ de la Constitución que establece el derecho a la educación primaria para todos los mexicanos, adicionando el principio de la obligatoriedad, con la finalidad de extender la educación elemental en los medios rurales y urbanos (Ordóñez, 2002). Es por esta razón que para materializar dicha estrategia se construyeron diversas escuelas en distintos lugares del país pero sobre todo en el área rural. De igual forma existió un importante apoyo al abasto de alimentos por medio de la intervención pública en los procesos de producción, distribución y consumo de alimentos básicos, lo cual generó que se pudiera contar con mayor cantidad de productos alimenticios tanto en áreas urbanas como rurales. Durante el cardenismo se lograron los cambios más trascendentales en el alivio a la pobreza dentro de lapso de los gobiernos posrevolucionarios.

En el periodo en que México empieza adoptar el modelo de sustitución de importaciones (1941), se observaron algunos avances en ciertas áreas del tratamiento hacia los pobres, como la creación en 1943 del Instituto Mexicano del Seguro social (IMSS) con la finalidad de ofrecer protección contra los riesgos de la vida y la vejez a amplios sectores de la población. Sin embargo, dicha estrategia se limitaba a las personas que tuvieran un trabajo formal. En el área de educación los progresos fueron insuficientes para resolver los problemas del analfabetismo y los rezagos en la cobertura de los servicios elementales, sobre todo entre los habitantes del campo. En este periodo se notó una clara tendencia por parte del gobierno en la inversión para lograr la industrialización, lo que tuvo algunos efectos negativos sobre los habitantes del campo, ya que la mayoría de los subsidios públicos se destinaron a los sectores medios populares de las ciudades en México 
(Ordóñez, 2002), dejando de lado la inversión en el campo mexicano. Posteriormente, esto ocasionó una emigración masiva del campo a la ciudad y por tanto el abandono de las tierras de cultivo.

Los resultados de casi todos los gobiernos posrevolucionarios reflejan que las estrategias estatales para proporcionar el bienestar social y mejorar la situación de pobreza pasaron a un segundo plano, ya que lo principal era el control militar y la pacificación del territorio. Es por esta razón que antes de 1950 no se puede distinguir la existencia de programas de combate a la pobreza como tal, por que el gobierno no reconocía aun las disparidades y desigualdades económicas que se estaban originando en México. Fue tan solo años después cuando el gobierno admitió los problemas de pobreza y marginación, estableciendo de esta forma las primeras estrategias para contrarrestar dichos problemas. Estas acciones fueron los distintos programas de alivio a la pobreza como el Programa de Inversiones para el Desarrollo Rural (PIDER), la Coordinación General del Plan Nacional de Zonas Deprimidas y Grupos Marginados (COPLAMAR), el Programa Nacional de Solidaridad (PRONASOL), el programa de educación, salud y alimentación (PROGRESA) y actualmente el de Oportunidades.

\section{DEL PIDER AL PROGRESA}

Las condiciones de marginación vividas desde la colonia, sumadas a la incapacidad de los primeros gobiernos posrevolucionarios para resolver los problemas de pobreza fueron sentando las bases para nuevas estrategias que permitieran el mejoramiento de las condiciones sociales. Es por esa razón que a partir de 1950 empiezan los primeros pasos contra la pobreza históricamente acumulada, lo que trajo un nuevo replanteamiento de las acciones del gobierno para el desarrollo del bienestar de la población. Durante este período, las etapas para contrarrestar este fenómeno se pueden dividir para su estudio en cuatro, que van desde los subsidios generalizados hasta el programa Oportunidades en el sexenio de Vicente Fox.

La primera, cubre el período entre los años cincuenta y setenta cuando el modelo económico en México era el de sustitución de importaciones. En el mismo, la superación de la pobreza descansaba primordialmente en el crecimiento de la economía, del empleo y los salarios reales (Cordera, 1999). Lo anterior permitió un crecimiento económico del sector industrial y de servicios, beneficiando a los trabajadores por medio de los subsidios 
indirectos a los salarios (SEDESOL, 2001) los cuales eran universales. Este acercamiento tenía la finalidad de mejorar las condiciones de vida de la población y por tanto de combatir la pobreza. La idea original era dotar de un mayor bienestar a los obreros comprometidos en el proceso productivo lo cual conduciría de manera más mediata a una expansión del bienestar en grupos más amplios de la población (Lerner, 1996). Por otro lado el sector rural jugó un papel importante, pues fue quien proveyó de bienes primarios y recursos naturales a bajo costo al sector industrial, mediante una amplia red de subsidios al campo, y una redistribución de tierra con el fin de reducir la subutilización de los recursos productivos.

En esta etapa se enfrentaron sinnúmero problemas, debido a que los subsidios para el bienestar, otorgados supuestamente de manera universal, tenian serias limitaciones que afectaban a los grupos más vulnerables. En específico, porque se otorgaban a través de las industrias, excluyendo a los que no estuvieran laborando en dicho sector. Por tanto, estos subsidios solo se materializaban para las personas que se encontraban laborando en las empresas; generando una mayor desigualdad económica al interior de las ciudades así como en el campo en donde el atraso era abismal, con lugares que no tenían la suficiente infraestructura para ser productivos. Aunado a esto existía una insuficiencia dinámica de la forma de crecimiento implantada en México la cual recogía una incapacidad del empleo para satisfacer necesidades de ingreso, así como en la cada vez más clara insuficiencia de las instituciones de seguridad y compensación social para incluir en sus beneficios a una población que crecía aceleradamente (Cordera, 1999).

En la búsqueda de nuevas fórmulas para corregir estos problemas, en 1973 el Estado mexicano con presidente Luis Echeverría inicia el primer programa de combate a la pobreza llamado Programa de Inversiones para el Desarrollo Rural (PIDER). Este programa se dirigió a regiones campesinas que se localizan en las zonas más atrasadas del país (localidades de más de 500 y menos de 5000 habitantes). De hecho el $40 \%$ de las acciones de este programa se orientaron a los estados de Jalisco, Oaxaca, Guerrero, Michoacán, Chiapas y Zacatecas, que tenían recursos pero carecían de infraestructura y servicios. El objetivo general del PIDER fue promover el desarrollo autosostenido de las comunidades rurales mediante la participación activa y organizada de sus habitantes, acción que unida a los esfuerzos del sector público permitirían generar excedentes económicos y 
mediante su inversión en óbras productivas, de apoyo y de bienestar social, lograrían un incremento sostenido del bienestar (Ordóñez, 2002).

El PIDER tenía como finalidad devolverle al sector agrícola el dinamismo que había perdido, sobre todo porque la pobreza rural crecía y no podía ser compensada con el reparto agrario y las personas del campo tampoco lograban ser absorbidas por el empleo urbano industrial. De esta forma se tenían que buscar vías de desarrollo intermedias o subalternas que dieran empleo y producción en las localidades rurales, para contrarrestar al mismo tiempo las tensiones sociales que habían empezado a renovarse en el campo (Cordera, 1999). El programa buscó de esta forma aumentar los ingresos y niveles de vida de los sectores rurales introduciendo actividades productivas que buscaban incrementar el empleo permanente y temporal (Villarespe, 2001).

En esencia estas acciones tenían un sustento lógico de acción pero en la práctica sucedió otra cosa de manera paradójica. Primero porque se generó un sesgo rural pues todos los beneficios se dirigieron al campo con la finalidad de compensar las desigualdades históricas entre ambos contextos (rural y urbano), dejando fuera de estos beneficios a un sinnúmero de personas que habían emigrado del campo a la ciudad y que habían formado los cinturones de miseria en las grandes urbes, como resultado del modelo económico de sustitución de importaciones y de los beneficios (subsidios) que los trabajadores tenían en estos lugares. Por otra parte, la esencia del programa sufrió desvíos pues los recursos destinados a la inversión en actividades productivas se utilizaron para caminos, electrificación, aulas, centros de salud, y agua potable, desvirtuándose de esta forma sus objetivos originales del fomento a la productividad rural como medio de mejoramiento de los ingresos y bienestar de los individuos en el campo. La consecuencia principal fue que la desigualdad en las grandes ciudades se incrementara paulatinamente, además que la emigración campo-ciudad siguió aumentando ante la falta de emplẹos que ayudaran al mejoramiento económico.

El gobierno de López Portillo tomando como referencia la experiencia del PIDER y sin desplazarlo del todo, propone en 1977 nuevas acciones por medio de la Coordinación General del Plan Nacional de Zonas Deprimidas y Grupos Marginados (COPLAMAR), cuyo objetivo general era agrupar y analizar las necesidades y las demandas más significativas de las diversas 
zonas marginadas, para que las diferentes entidades de la administración pública pudieran concurrir como un todo coherente (COPLAMAR,1982:21).

El COPLAMAR orientó sus acciones en dos direcciones: a) influir sobre las causas generadoras de la depresión económica y la marginación; b) actuar sobre los efectos más graves que han dejado como secuela una marginación de centurias y que se manifiesta en el enorme déficit existente en las áreas rurales en materia de alimentación, salud, educación, equipamiento comunitario, infraestructura de apoyo a la producción y otros servicios colaterales. La población a quien se dirigió el programa eran los indígenas y campesinos pobres que habitaban en 30 mil localidades dispersas en el desierto, semidesierto, pantanos y zonas montañosas del país (Ordóñez, 2002), considerados como marginados del desarrollo.

El COPLAMAR a pesar de que tenía una visión clara hacia donde dirigirse (al menos en sus comienzos), tuvo como problema central (al igual que el PIDER) el tener un sesgo eminentemente rural, lo cual descuidó la pobreza urbano-marginal. Sin embargo, sus mejores resultados se dieron cuando trabajó con entidades cuya dinámica y presencia institucionales resultaban sólidas (Pardo, 2000), como por ejemplo el IMSS-COPLAMAR y Diconsa, al que se une el Sistema Alimentario Mexicano (SAM) que pretendía darle estatuto de estrategia a la idea de autosuficiencia alimentaria (Cordera, 1999).

Los dos programas señalados, a pesar de ser parecidos tuvieron diferencias sustanciales. El COPLAMAR a diferencia del PIDER, nunca tuvo intención de construir proyectos que se autofinanciaran en un determinado plazo pero si involucraba la formación de comités integrados con la población a ser beneficiada, ello obedecía más bien a las necesidades de la dependencia que llevaría a cabo la obra (Villarespe, 2001). A pesar de que tanto el PIDER como el COPLAMAR buscaron reducir las brechas de la pobreza en la década de los setenta (ya que fueron contemporáneos después de 1977) los resultados fueron mínimos; incluso a finales de esa década la política social se le equipara con una especie de ambulancia que recogía a los heridos por los programas de ajuste, pero los cuales no tenían posibilidades de cura alguna (Pardo, 2000). Esto generó por parte del gobierno el replanteamiento sobre la viabilidad de sus estrategias de combate a la pobreza.

Al iniciar los primeros años de la década de los ochenta, México empieza a vivir una serie de cambios económicos y sociales, que redundaron en 
problemas para los grupos más vulnerables, pues en ese momento se adoptó el modelo económico neoliberal en un contexto marcado por una drástica caída en los precios del petróleo (1982) y una crisis que puso al país en una recesión económica junto con una creciente inflación y devaluación del tipo de cambio así como una contracción del producto interno bruto (SEDESOL, 2001). Es de esta forma que el cambio de modelo de desarrollo económico trajo como resultado el aumento de la pobreza, sumado a esto se cerraron medianas y pequeñas empresas que no pudieron competir ante productos importados, además del adelgazamiento del Estado que provocó el despido de burócratas (Lerner, 1996). Como resultado, el gasto social se recorta y por tanto el sistema de subsidios se desmanteló y los programas de combate a la pobreza no fueron prioridad para el gobierno.

Las medidas adoptadas en el periodo del presidente Miguel de la Madrid repercutieron en una mayor desigualdad social pues no se invirtió en los grupos marginados, ya que en ese sexenio fueron desmantelados la mayoría de los programas de alimentación creados por López Portillo y no existió una agenda específica de combate a la pobreza, es decir, no hubo un programa que enviara recursos hacia las zonas más marginadas del país (Favela y otros, 2003). Hemos denominado a este lapso como el periodo muerto de los programas de combate a la pobreza en México, pues en éste periodo los recursos para este fin eran casi nulos. No obstante, la estrategia que debió haberse aplicado tendría que haber sido la contraria, ya que en tiempos de inflación la mejor estrategia para compensar este fenómeno y que la gente pobre pueda sobrevivir es mediante el incremento del gasto social.

La situación de pobreza que se vivía en el país a finales de los 80 's, originó que el gobierno reconociera que los grupos más desfavorecidos de la sociedad eran quienes más habían sufrido con el estancamiento de la economía. Durante el sexenio de Carlos Salinas de Gortari se diseñó y puso en práctica el Programa Nacional de Solidaridad (PRONASOL), iniciando de esta forma la segunda etapa de los programas de combate a la pobreza. El PRONASOL fue visto como un instrumento renovador de la política social capaz de corregir las desviaciones del viejo estado posrevolucionario, que según la versión oficial se había caracterizado por su perfil burocrático, paternalista, centralizado y corporativo (Ordóñez, 2002). Por tanto, dicho programa tuvo como finalidad combatir los efectos que las políticas económicas y la crisis tuvieron sobre las personas más pobres, de ahí que su población objetivo fueron los pueblos indígenas, campesinos, habitantes del 
medio rural, trabajadores agrícolas migratorios, habitantes de colonias populares de las ciudades, jóvenes, mujeres y niños (Ordóñez, 2002). Como punto importante dentro de la inclusión de sus destinatarios fue la incorporación de los habitantes de las colonias populares de las ciudades así como los jóvenes, mujeres y niños, considerados como grupos prioritarios a diferencia de los programas que lo antecedieron.

El PRONASOL se regía por cuatro principios básicos: a) respeto a la voluntad, iniciativa y formas de organización de los individuos y comunidades; b) participación y organización plena y efectiva de las comunidades; c) corresponsabilidad social; d) transparencia, honestidad y eficiencia en el manejo de los recursos (Villarespe, 2001). De ahí que sus objetivos fueron la creación de las condiciones físicas y socioeconómicas necesarias para la igualdad de oportunidades; el pleno desarrollo de las capacidades de la población; la apertura de espacios de decisión a la participación social organizada de las comunidades y el efecto redistributivo del gasto social a través de una mejor selección de los beneficiarios. El programa planteó primeramente como medio para combatir la pobreza, el cubrir los rezagos en materia de bienestar, además de generar la infraestructura necesaria para potenciar la economía de las regiones más atrasadas del país. Una vez que se solucionaran estos aspectos básicos del bienestar, el siguiente paso consistiría en la creación de empleos productivos e ingresos fijos en las comunidades (Ordóñez, 2002). El programa se enfocó a la construcción de infraestructura física y social, en donde la organización y la participación ciudadana (a través de los comités de solidaridad) fueron requisito indispensable para poder participar y beneficiarse del programa y con ello proporcionar una red de seguridad para los segmentos más frágiles de la sociedad.

El PRONASOL contaba con dos características que lo hacían fundamentalmente distinto al PIDER y al COPLAMAR. La primera fue que se basaba en el supuesto que cuando se tienen recursos escasos, una opción para maximizar el impacto de los programas es canalizar beneficios exclusivamente hacia la población objetivo (aunque esto solo sucedió desde el discurso), es decir se le concibió como una política compensatoria dirigida a grupos específicos. La segunda característica fue que el programa tenía como uno de sus principios básicos la participación social, en el que las comunidades participaban desde el diseño hasta la evaluación y mantenimiento de las obras. La cooperación de las comunidades en sus obras se realizaba mediante trabajo directo, entregando recursos y materiales 
propios de la región (SEDESOL, 2001). Desde el discurso gubernamental la participación de la comunidad era un factor importante para el éxito del programa, pero en la realidad se limitaba a la asistencia a las asambleas y en el mejor de los casos a trabajar en la realización de las obras, debido a que las obras y proyectos estaban ya etiquetados, por lo que si se gestionaba una obra no considerada en el catálogo ésta no se podía realizar, además que los criterios de focalización nunca estuvieron claros, de ahí que los recursos se destinaban según las necesidades políticas del gobierno federal.

El PRONASOL tuvo algunos éxitos en ciertas áreas del combate a la pobreza, principalmente en la dotación de infraestructura básica en zonas con un alto grado de marginación, pero tuvo varios problemas debido a que se concentró en la pobreza moderada, básicamente urbana y careció de una estrategia específica para aliviar la extrema, principalmente la rural (Favela y otros, 2003). Una de las explicaciones al respecto fue por la forma de focalización de los recursos ya que no existía una metodología clara y por tanto los recursos se destinaron hacia las zonas urbanas o en los estados donde el gobierno salinista había tenido problemas en las elecciones, descuidándose de esta forma distintos contextos. Una de las problemáticas de corte político y normativo que tuvo fue que se le consideró como un programa presidencialista exento de vigilancia por parte de las Secretarías encargadas del programa. La evaluación no se contemplaba, por tanto la asignación de recursos antes que responder a la lógica de combate a la pobreza fue orientada con fines político-electorales, pues se buscaba consolidar el gobierno salinista debido a los problemas que tuvo en la legitimación de su triunfo en las votaciones.

La tercera fase de los programas de combate a la pobreza inicia en la segunda mitad de los años noventa durante el gobierno de Ernesto Zedillo, donde primeramente se da un cambio en la ideología sobre la pobreza, pues se consideró que es un problema que afecta la capacidad del individuo para generar de manera permanente el ingreso que le permita satisfacer sus necesidades básicas (Favela y otros, 2003). Es por ello que la política de superación a la pobreza se orientó a la aplicación de acciones concretas en educación, salud, alimentación y vivienda, dirigida a grupos de alta vulnerabilidad para mejorar sus condiciones de vida, aprovechando la organización social y ciudadana existente, pero sobre todo para formar capacidades básicas en la población. Instrumentándose de esta forma nueve líneas de acción las cuales fueron: 
1. Política de formación de capital humano: a) Programa de Educación, Salud y Alimentación (PROGRESA).

2. Políticas asistenciales: a) programa de subsidio a la tortilla mediante, el cual consistía en el otorgamiento de un kilo de tortillas a las familias con ingresos menores a dos salarios mínimos, áreas urbanas y semi-urbanas; b) programa de abasto social de leche (Liconsa) vendiéndola a menos de la mitad del costo en cualquier otra tienda; c) programa social de abasto (Diconsa) en el cual se podían adquirir productos básicos a precios más bajos que cualquier otro establecimiento.

3. Políticas de formación de capital familiar: a) vivienda progresiva: se buscó otorgar lotes en zonas urbanas. b) vivienda rural: se apoyó la autoconstrucción así como el otorgamiento de material para los techos de las viviendas.

4. Política de desarrollo de infraestructura social básica: atiende lo relacionado con el grado de acceso a los servicios públicos básicos de comunidades rurales especialmente en la construcción de sistemas de electrificación, agua potable, alcantarillado, drenaje, en el cual los recursos se otorgaban principalmente por los fondos de infraestructura social.

5. Políticas de impulso al desarrollo productivo: empleo temporal, crédito a la palabra, cajas solidarias, programa nacional con jornaleros agrícolas, programa de mujeres, desarrollo de pueblos indígenas, programa de la comisión nacional de zonas áridas, fondo nacional de artesanías, fondo nacional de apoyo a empresas sociales. Su finalidad era hacer productivo el campo mediante diversos subsidios y apoyos al mismo.

6. Políticas de desarrollo de infraestructura básica: se orientó principalmente a la construcción de de carreteras y puentes que permitiera un mejor traslado de las comunidades más alejadas.

7. Políticas de desarrollo de canales de comercialización, cadenas productivas y programas de capacitación; se instrumentó conjuntamente con la Secretaría de Comercio con la finalidad de que las comunidades pudieran vender a mejores precios sus productos locales.

8. Políticas de desarrollo regional: atendió la detección de los recursos regionales y su posible utilización productiva. 
9. Políticas de promoción de la participación ciudadana en el desarrollo social: en todas estas políticas se asume que la participación social es un ingrediente insustituible en el éxito de la estrategia de desarrollo social y combate a la pobreza (Villarespe, 2001).

Dentro de estas líneas de acción para el combate a la pobreza la estrategia principal fue el Programa de Educación, Salud y Alimentación (PROGRESA), el cual estaba dirigido a las familias que vivían en condición de pobreza extrema en las áreas rurales, con el fin de ampliar y mejorar las oportunidades de educación, salud y nutrición. Estos componentes buscaban elevar las capacidades de sus miembros para alcanzar mejores niveles de bienestar (Congreso de los Estados Unidos Mexicanos, 2002). A diferencia del PRONASOL los objetivos del PROGRESA estaban delimitados pues se concibió al programa como un medio para hacer frente a los costos sociales de la crisis económica de los años ochenta, las políticas de ajuste así como reestructurar las relaciones entre el Estado y la sociedad. De igual forma buscaba enfrentar la pobreza perpetuada por generaciones al romper con su círculo intergeneracional, de esta forma sus objetivos centrales fueron:

1. Mejorar las condiciones de alimentación, salud y educación, en particular de los niños y de sus madres, para un mejor aprovechamiento escolar y abatir la deserción escolar.

2. Apoyar la economía familiar, procurando que el hogar disponga de recursos suficientes para que los hijos completen su educación básica.

3. Hacer co-responsables y partícipes a todos los miembros de la familia en acciones de bienestar social.

4. Promover la participación y el respaldo comunitario a las acciones que se emprendan, para que los servicios educativos y de salud beneficien al conjunto de las familias (Congreso de los Estados Unidos Mexicanos, 2002).

En otras palabras, el programa buscó eliminar los obstáculos que impedían a las familias pobres el acceso a niveles suficientes de nutrición y salud, así como desarrollar sus capacidades para formar un capital humano. Para lograrlo utilizó tres componentes. El primero fue el educativo que consistía en becas y apoyos en especie o en dinero para útiles escolares, siempre y cuando haya una asistencia regular de los niños a la escuela, con becas desde 
tercer año de primaria hasta tercero de secundaria, siendo ligeramente mayores para las niñas en relación con los niños, con la finalidad de que no abandonaran los estudios a temprana edad como sucedía en los hogares de extrema pobreza. Con este componente se pretendió dotar a los individuos de oportunidades para su formación integral y mejores capacidades para su posterior ingreso al mercado laboral.

El segundo componente fue la salud, que consistió en un paquete básico que integra pláticas para el cuidado de la salud así como elementos preventivos de enfermedades, partiendo del supuesto que al mejorar dicho aspecto (salud) podría incrementarse la productividad en la escuela o trabajo, permitiendo no dejar de acudir a dichos lugares. El tercer componente, fue la alimentación para la cual se otorgó dinero en efectivo para ser utilizado para lograr una mayor calidad alimentaria, de igual forma se entregaban suplementos alimenticios con micronutrientes para las mujeres embarazadas y en periodo de lactancia así como a los niños que se detectaron con problemas de desnutrición, con la finalidad que ambos grupos vulnerables consumieran los requerimientos mínimos de calorías (Congreso de los Estados Unidos Mexicanos, 2002).

En cuanto a la asignación de los beneficios del programa se utilizó la focalización, el cual no es un concepto nuevo en las políticas sociales en el mundo ya que su objetivo es atender con precisión la satisfacción de necesidades de personas excluidas de satisfactores mínimos o esenciales (la población objetivo más vulnerable), por razones culturales, socioeconómicas y de costos de transacción (Contreras, 2000). El PROGRESA de esta forma a diferencia de los programas anteriores fue uno de los primeros en utilizar una metodología científica para localizar a las comunidades y familias beneficiadas. Se parte de la idea que por medio de este proceso se pueden atender con precisión a los beneficiarios potenciales. De ahí que para la identificación de las familias beneficiadas utilizaba dos procesos:

El primero a nivel macro, se utilizaba un índice calculado por el Consejo Nacional de Población (CONAPO) para ubicar las zonas de alta marginación en el país. Sin embargo, es posible que por medio de la focalización varios hogares pobres que no se encuentran en las localidades consideradas de muy alta marginación o alta marginación sean excluidos. Además si una comunidad era señalada para ser beneficiada del programa pero si esta no contaba con la infraestructura necesaria (escuela y centro de salud cercanos) para que el programa operara este no se aplicaba, dejando entonces fuera a 
los lugares que debido a su inaccesibilidad no tenían la infraestructura necesaria para la operatividad del programa lo cual era un común denominador en las zonas rurales de mayor pobreza.

La segunda focalización es a nivel micro la cual consistía en identificar dentro de la comunidad a las familias que por sus carencias eran elegibles a ser beneficiadas del programa, aplicándoles una encuesta para conocer su nivel socioeconómico. A partir de esos datos obtenidos se utilizaba el análisis discriminante que es técnica estadística multivariable, permitiendo identificar a los hogares que tuviera las mayores necesidades y por ende ser beneficiado. A pesar de la utilización de dicha metodología Escobar y González de la Rocha (2002) encontraron que existían personas que no habían sido beneficiadas las cuales tenían iguales o mayores carencias de las que si recibían el programa. Con todo lo anterior es necesario recalcar que a pesar de los problemas de esta metodología se sigue utilizando por los programas actuales de combate a la pobreza en México (como es el Oportunidades).

Desde el discurso del gobierno el PROGRESA era considerado como un programa de combate a la pobreza y no tanto de inversión en capital humano que buscara romper el círculo intergeneracional de la pobreza al dotar de las herramientas necesarias (educación, salud y alimentación) para mejorar sus condiciones sociales, sin embargo esta postura fue poco promocionada en esta etapa y fue hasta la siguiente con el programa Oportunidades que se hizo énfasis en esta posición.

\section{El Programa Oportunidades}

La cuarta etapa de los programas de combate a la pobreza tiene una relación directa con la alternancia política del año 2000, debido que a partir de 2002 el PROGRESA se transforma en el Programa de Desarrollo Humano Oportunidades. El cual vincula en su operación a las Secretarías de Desarrollo Social, Educación Pública y Salud, así como el Instituto Mexicano del Seguro Social en coordinación con las autoridades sectoriales estatales, con la finalidad de proporcionar en forma adecuada los servicios de educación y de salud, la capacitación de su personal y la certificación de las corresponsabilidades de las familias, en el marco de los acuerdos establecidos entre el Gobierno Federal y los Gobiernos Estatales. Mediante dicha coordinación aunada a las estrategias propuestas se busca romper el 
círculo intergeneracional de la pobreza mediante la inversión en capital humano, de ahí que se plantea como objetivo general:

Apoyar a las familias que viven en condición de pobreza extrema con el fin de potenciar las capacidades de sus miembros y ampliar sus alternativas para alcanzar mejores niveles de bienestar, a través del mejoramiento de opciones en educación, salud y alimentación, además de contribuir a la vinculación con nuevos servicios y programas de desarrollo que propicien el mejoramiento de sus condiciones socioeconómicas y calidad de vida (SEDESOL, 2005:6).

El objetivo planteado muestra como el programa se centra en la inversión en capital humano mediante el desarrollo de capacidades y acciones intersectoriales coordinadas de educación, salud y alimentación. La forma en como pretende lograrlo es mediante la asistencia escolar, incrementos en la utilización de los servicios de salud para el cuidado preventivo y mejorando el estado nutricional de los beneficiados (Coady, 2000). Todo ello a través de tres componentes básicos los cuales trabajan de forma coordinada y que son:

a) Componente educativo: Integrado por becas las cuales están dirigidas a cada uno de los niños, niñas y jóvenes de los hogares beneficiados, siempre y cuando estén inscritos en grados escolares comprendidos entre tercero de primaria y tercero de nivel medio superior ${ }^{3}$. Este dinero se entrega durante los diez meses del ciclo escolar, su monto es creciente a medida que avanzan de grado escolar, además que en secundaria y educación media superior las becas para las mujeres son superiores a las de los hombres, con el fin de compensar que en las familias en condición de pobreza extrema son ellas quienes tienden a abandonar sus estudios en mayor proporción y en edades más tempranas. Los becarios de primaria además reciben al inicio del ciclo escolar un apoyo monetario para adquirir útiles escolares o el paquete en si (de acuerdo con las normas del Consejo Nacional de Fomento Educativo) y al inicio del segundo semestre del ciclo escolar se les otorga un apoyo monetario

\footnotetext{
${ }^{3}$ Existe un requisito en cuanto a las edades los niños que cursan entre tercero de primaria y tercero de secundaria deben ser menores de 18 años y los que se encuentren cursando el nivel medio superior deben de tener una edad entre 14 y 20 años.
} 
adicional para la reposición de útiles escolares, mientras los becarios de secundaria y de educación media superior, reciben un único apoyo monetario anual para la adquisición de útiles escolares el cual se entrega durante el primer semestre del año (SEDESOL, 2005).

b) Componente de salud: Opera bajo cuatro estrategias específicas: a) Proporcionar de manera gratuita el paquete esencial (básico) de Servicios de Salud; b) Prevenir la desnutrición de los niños desde la etapa de gestación mediante la entrega de suplementos alimenticios; c) Fomentar y mejorar el autocuidado de la salud de las familias y de la comunidad a través de la comunicación educativa en materia de salud, nutrición e higiene y; d) Reforzar la oferta de servicios de salud para satisfacer la demanda adicional. Estas estrategias se dirigen con especial énfasis, a la población más vulnerable como las mujeres embarazadas y en periodo de lactancia, y los niños menores de cinco años.

c) Componente alimentario: Consiste en apoyos monetarios directos a las familias beneficiarias para contribuir a que mejoren la cantidad, calidad y diversidad de su alimentación $\mathrm{y}$, por esta vía, elevar su estado de nutrición, asimismo se entrega suplementos alimenticios que buscan reforzar la alimentación infantil y de las mujeres embarazadas y/o en periodo de lactancia (Congreso de los Estados Unidos Mexicanos, 2002).

El programa Oportunidades al igual que el PROGRESA destina los recursos a las personas con mayor grado de marginación, mediante un proceso de doble focalización. La primera es la selección de zonas altamente marginadas, en el caso de las rurales se basa en los niveles de marginación establecidos por el CONAPO. En el caso de las zonas urbanas se utiliza la información estadística disponible a nivel de localidades, áreas geoestadísticas básicas (AGEBs), colonias o manzanas, generada por el Instituto Nacional de Estadística, Geografía e Informática (INEGI), dando prioridad a la selección y atención de aquellas donde la concentración de hogares en pobreza extrema, con este proceso se busca identificar los agrupamientos geográficos en los cuales se pueda alcanzar los mayores beneficios para los hogares (Congreso de los Estados Unidos Mexicanos, 2002). Una vez seleccionadas las zonas de concentración, se procede a validar las condiciones de accesibilidad y capacidad de atención de los servicios de salud y educación que permitan operar en forma integral los tres componentes del programa (SEDESOL, 2005), lo que puede resultar en la 
exclusión de familias que no se encuentren viviendo en la zona detectada como prioritaria aunque sus características socioeconómicas pueden ser iguales o peores que las personas que viven en áreas consideradas como marginadas.

La segunda focalización pretende la identificación de las familias en condiciones de pobreza extrema en las zonas señaladas como prioritarias, esto lo realizan mediante un criterio que la SEDESOL argumenta que es homogéneo, objetivo y único en todo el país tanto en zonas urbanas y rurales. Para lograrlo se aplica una cédula de identificación socioeconómica, donde se recopilan datos sobre las características del hogar y de las condiciones socioeconómicas de todos sus miembros. La cédula esta integrada por los factores que intervienen en la determinación de la condición de pobreza extrema, que fueron definidos a partir del análisis detallado de fuentes de datos socioeconómicos de los hogares con representatividad nacional. Dichos factores son: estructura del hogar, características individuales, ocupación, ingresos de los miembros del hogar, apoyos gubernamentales, créditos, emigración de integrantes del hogar, servicios de salud y miembros discapacitados del hogar, características de la vivienda. Con los datos obtenidos se aplica el análisis discriminante el cual resume la información de los hogares en un solo indicador que es una medida de la condición de pobreza extrema del hogar (Congreso de los Estados Unidos Mexicanos, 2002) el dato generado permite determinar que hogares son los factibles de ser beneficiados.

El procedimiento anterior puede excluir familias al no llenar correctamente la cedula de identificación, ya sea por no conocer bien los datos, por pena, por no entender bien las preguntas o simplemente porque así considera que le darán el apoyo del programa. Ante esa situación es necesario analizar los mecanismos de focalización del programa, ya que dentro de las comunidades que de antemano son marginadas el seleccionar a unas cuantas unidades domésticas para ser beneficiadas puede generar un desgaste del tejido social, al generar una selectividad entre unos y otros, sobre todo cuando las características de pobreza son similares entre ellos, afectando de esta forma las relaciones al interior de la comunidad, pues los programas focalizados no logran unir a las comunidades sino más bien las dividen en tanto crean distinciones entre los que son beneficiados y los que no lo son (Lerner, 1996). En esa misma línea Escobar y González de la Rocha (2002) afirman: 
En casi todas las localidades en estudio, se encontró una cantidad apreciable de hogares excluidos del programa cuyos ingresos domésticos eran, en términos generales, comparables o inferiores a los de hogares incluidos, dichos hogares no se distinguían tampoco por poseer mejores viviendas o menores índices de dependencia económica (pág. 45).

Escobar y González de la Rocha (2002) mencionan que en sus investigaciones no encontraron evidencias sobre diferencias entre los hogares beneficiados y los no beneficiados en términos de ingresos, ya que algunos de los hogares que reciben el programa tienen ingresos más altos tanto a nivel doméstico como per cápita, a diferencia de los hogares que no recibían apoyos del programa. Por tanto el proceso de focalización puede tener posibles exclusiones a partir de la distorsión de la información porque hay personas que no declaran todos sus ingresos económicos en el momento que se le aplica los estudios socioeconómicos, provocando que no recibieran el programa algunas familias que si declararon los datos de manera correcta sobre todo los ingresos, teniendo como resultado final el incluir en los necesitados a los que no lo son (error tipo I). Por otra parte puede suceder que algunas familias pobres hayan declarado mayores ingresos debido al estigma al interior de la comunidad por declararse como pobres, ya que repercutiría en su amor propio (Sen, 2000), falseando de esta forma la información sobre su situación real.

Lo anterior lleva a señalar que el programa Oportunidades debería tener ciertas modificaciones en cuanto a la selección de beneficiarios, tal vez seguir siendo focalizado a nivel macro (para dirigirlo a los contexto con mayor pobreza) pero universal a nivel micro (dentro de las comunidades), para que las familias que viven en las zonas de antemano detectadas de pobreza extrema puedan recibir todas los beneficios sin caer en una exclusión. Es decir debería situarse entre los extremos constituidos por la focalización perfecta y por la universalidad completa. Sin embargo, a partir del 2005 la prioridad del programa no es el seguir ampliando la cobertura, sino la sustitución de las bajas del padrón para mantener la cobertura de atención en 5 millones de familias beneficiarias (SEDESOL, 2005), lo que significa que la inclusión de más hogares ya no es prioridad, sino ahora se pretende desde el discurso mejorar los servicios de las familias que reciben el programa, manteniendo el número actual. 
Lo hasta ahora visto en relación al programa Oportunidades lleva a cuestionarse sobre ¿Cuál es el sustento teórico atrás de dicha estrategia del gobierno? La respuesta a dicha interrogantes es que se basa en la teoría de capital humano, de ahí que se tipo de programas a nivel mundial se llaman programas de transferencias condicionadas (CCT) a la formación de capital humano, los cuales se han implementado en distintos países en vías de desarrollo. La razón principal de la aplicación de este tipo de estrategias no solo es por el ingreso que las familias pueden obtener, sino también porque el desarrollo de los países se ha basado en un claro entendimiento de la importancia que tiene la inversión en seres humanos como factor competitivo y de progreso (Ramírez, 1999). De igual forma Sen (2000:162) señala que "los países que hoy son ricos poseen una historia bastante notable de medidas públicas relacionadas con la educación, la asistencia sanitaria, la reforma agraria", de tal forma que estas estrategias sociales permitirán a la mayoría de los individuos participar directamente en el proceso de expansión económica y de esta forma compensar la elevada desigualdad de activos generadores de ingresos y con ello reducir la pobreza.

Los argumentos de la teoría de capital humano que sostienen al programa Oportunidades hacen cuestionar varios aspectos. Primero, porque en su componente de educación se argumenta que al tener el bachillerato (que es el nivel máximo que cubre el programa) las personas podrán romper el círculo intergeneracional de la pobreza al insertarse en el mercado laboral. Sin embargo, las retribuciones monetarias (salariales) que se logran por alcanzar este nivel no son significativas y no hace la diferencia en términos económicos ya que desde finales de los 60 's se ha comprobado que los beneficios reales se observan al tener una licenciatura (Becker,1993), cosa que a más 40 años de esas afirmaciones parecerían obsoletas por el incremento de personas con títulos universitarios y si a eso le sumamos la escasez de empleos o autoempleos en México, dificulta aún más salir de esas condiciones económicas desfavorables.

El segundo aspecto que cabe cuestionarse es que estos programas buscan romper con el costo de oportunidad de las familias de mandar a trabajar a sus hijos y en lugar de eso asistan a la escuela e incrementar su capital humano. De ahí que las transferencias económicas (becas) tienen esa finalidad al motivar a los beneficiarios a seguir estudiando solventando los costos directos que se generan de ese gasto. Sin embargo, pudiera no tener el impacto esperado debido a que los montos que se obtienen son menores a los 
que se reciben en la familia por el trabajo de un niño o joven de las edades que cubre el programa. Por tal razón, no resultaría atractivo el seguir estudiando y optar mejor por integrarse a la vida laboral. Es importante mencionar que los impactos de ese tipo de programas son a largo plazo y por tanto sus "resultados se obtendrán en los próximos diez o veinte años" (Heckman, 2004:3) que será cuando los rendimientos de la inversión serán parte de las retribuciones (Becker, 1993). Para entonces los beneficiarios estarán insertados en el mercado de trabajo, por lo que ver los beneficios reales de este programa en el aspecto de pobreza es prematuro pues únicamente se puede observar la acumulación de capital humano que va generando en sus beneficiarios.

Otro aspecto a cuestionar es que la teoría del capital humano se hace dependiente del mercado mismo y por tanto también el programa. De ahí que al no tener las suficientes fuentes de empleo o autoempleo que absorban a las personas en que se esta invirtiendo será un gran problema a corto plazo, trayendo como resultado que el círculo intergeneracional de la pobreza no se rompa y en el mejor de los casos los beneficiarios en quienes se invirtió tengan que emigrar para conseguir un trabajo. Finalmente, si bien es cierto que el programa Oportunidades esta invirtiendo en salud y alimentación, esenciales para el desarrollo de capital humano, aquí intervendrán para su óptimo resultado la calidad de los servicios y los montos de las transferencias para adquirir una adecuada alimentación. Aun así es importante señalar que la educación, la salud y la nutrición no pueden por sí solas transformar la economía. Por lo que son necesarios otros elementos para la creación del bienestar, especialmente fuentes de empleo ya que en la realidad mexicana este punto es uno de los más débiles actualmente, por lo que los programas de combate a la pobreza tienen que vincularse a una política laboral para que realmente estén a tono con las necesidades sociales del país.

\section{Conclusión}

El tratamiento de la pobreza en México ha pasado por diversas etapas en las cuales se han ido mejorando las acciones a base de prueba y error, donde sin duda se han logrado en cada momento histórico diversos beneficios para los grupos más vulnerables. Por tanto responder a la pregunta si ¿los programas de alivio a la pobreza en México han respondido a las necesidades sociales que se vivían en los distintos momentos históricos en los que se aplicaron? 
Podemos señalar que desde la época colonial con los asilos y hospicios hasta la actualidad con los programas de inversión en capital humano, han sido considerados como las estrategias más ideales en cada época, aun cuando no han resuelto el problema por el que fueron creados. Sin embargo en todo este tiempo en las estrategias se han tenido una serie de limitaciones, en algunos casos por la falta de una clara metodología y en algunos otros por la falta de un concepto claro que delimitará a quienes se tenían que enfocar las acciones, de esta forma se ha dejado un asunto inconcluso en materia de pobreza y desarrollo humano en México.

Lo anterior lleva a reconocer que las estrategias emprendidas en contra de la pobreza por parte del gobierno no han respondido a las necesidades reales de la época, por lo que se puede decir que los cambios y necesidades sociales se dan de una manera más acelerada que las mismas acciones que buscan contrarrestarlas. Esto también sucede porque las acciones en contra de este fenómeno se han dado mediante ayudas mediáticas que fuera de contrarrestar la pobreza de forma estructural solo han buscado una ayuda a corto plazo, por tanto las acciones gubernamentales únicamente se han enfocado en atacar los síntomas sociales más evidentes y no los problemas de fondo que dan origen a la pobreza y marginación.

Lo que es importante reconocer es que ahora con las estrategias de inversión en capital humano se busca romper con el círculo intergeneracional de la pobreza con una visión a largo plazo, al dotar de mayor educación, salud y mejor calidad de nutrición a sus beneficiarios. Claro que el logro de esta política solo puede tener frutos si es acompañada de espacios laborales para que las personas en quienes se invierte puedan integrarse a empleos o autoempleos. En caso contrario ¿qué sucedería en un tiempo no muy lejano cuando los hijos de los 5 millones de familias beneficiadas del programa Oportunidades pretendan integrarse a la vida laboral y no existan espacios suficientes para ellos? Es probable que emigren a los Estados Unidos, mejorando la calidad en la formación de migrantes que estarán mejor capacitados y con mayor nivel educativo, representando una fuga importante de los recursos públicos que no tendrían impacto en el desarrollo de la economía nacional.

Finalmente es importante aclarar que los programas de combate a la pobreza son una estrategia prioritaria para el mejoramiento de las condiciones sociales de las familias, por tanto es necesario no desvincularlas de la realidad actual y las necesidades mismas de la sociedad. 


\section{Referencias}

Becker, Gary. (1993). Human Capital: A theoretical and empirical analysis, with special reference to education. Chicago: University of Chicago Press.

Coady, David. (2000). La aplicación del análisis social de costo-beneficio a la evaluación de PROGRESA. En: Evolución de resultados del programa de educación, salud y alimentación, Análisis de costo beneficio. México D.F.: Secretaría de Desarrollo Social.

Congreso de los Estados Unidos Mexicanos. (2002, 15 de marzo). Acuerdo que establece las Reglas de Operación del Programa de Desarrollo Humano Oportunidades para el ejercicio fiscal. Diario Oficial de la Federación. México: Secretaría de Desarrollo Social.

Congreso de los Estados Unidos Mexicanos. (2004, 20 de enero). Ley General de Desarrollo Social. Diario Oficial de la Federación. México: Secretaría de Desarrollo Social.

Congreso de los Estados Unidos Mexicanos. (2005). Programa de Desarrollo Humano Oportunidades, reglas de operación para el ejercicio fiscal 2005. México, D.F: Secretaría de Desarrollo Social.

Contreras, Enrique. (2000). El diseño administrativo de los programas de emergencia. En R. Cordera y A. Zicardi (coordinadores), Las políticas sociales de México al final del milenio descentralización, diseño y gestión, 497-4510. México: Ed. Porrua.

Coordinación General del Plan Nacional de Zonas Deprimidas y Grupos Marginados. (1982). Memoria de actividades 1976-1982. COPLAMARpresidencia de la republica. México: Autor.

Cordera, Rolando. (1999). PROGRESA y la experiencia mexicana contra la pobreza. En: J. Gómez y R. Loyola (Comps.), Alivio a la pobreza: Análisis del programa de Educación, Salud y Alimentación, 13-23. 
México: Centro de Investigaciones y Estudios Superiores en Antropología social.

Escobar, Agustín y González de la Rocha, Mercedes. (2002). Documento final de diagnóstico cualitativo de hogares semi urbanos en localidades de 2500 a 50000 habitantes. México: Centro de Investigaciones y Estudios Superiores de Antropología Social

Favela, Alejandro y otros. (2003). El combate a la pobreza en el sexenio de Zedillo. México: Editorial Plaza y Valdés.

Heckman, James. (2004). Inversión en capital humano y pobreza. Argentina: Centro de Evaluación y Estudios en Economía Social para el Alivio de la Pobreza (CESyP), Universidad Torcuatto di Tella.

Lerner, Bertha. (1996). América Latina: los debates en política social, desigualdad y pobreza. México: Editorial Porrua.

Ordóñez, Gerardo. (2002). La política social y el combate a la pobreza en México. México: Centro de Investigaciones Interdisciplinarias en Ciencias y Humanidades de la Universidad Nacional Autónoma de México.

Pardo, Maria del Carmen. (2000). El diseño administrativo de los programas de emergencia. En R. Cordera y A. Zicardi (coordinadores), Las politicas sociales de México al final del milenio descentralización, diseño y gestión, 459-479. México: Ed. Porrua.

Ramírez, Hugo. (1999). El capital intelectual, base de la capacidad competitiva de la organización. Revista de comercio exterior; 49 (12), 1047-1060.

Secretaría de Desarrollo Social. (2001). Programa Nacional de Desarrollo Social 2001-2006, Superación de la pobreza: una tarea Contigo Primera edición. México, DF: Autor.

Secretaría de Desarrollo Social. (2003). Programa Institucional Oportunidades 2002-2006. México, DF: Autor. 
Secretaría de Desarrollo Social. (2005). Programa de Desarrollo Humano Oportunidades, reglas de operación para el ejercicio fiscal 2005. México, DF.: Autor.

Secretaría de Desarrollo Social. (2006). Programa de Desarrollo Humano Oportunidades, reglas de operación para el ejercicio fiscal 2006. México, DF: Autor.

Sen, Amartya. (2000). Desarrollo y Libertad. México: Editorial Planeta.

Villarespe, Verónica. (2001). La solidaridad: beneficencia y programas. Pasado y presente del tratamiento de la pobreza en México. México: Textos Breves de Economía del Instituto de Investigaciones Económicas de la Universidad Autónoma de México (primera edición).

Villatoro, Pablo. (2004). Programas de reducción de la pobreza en América Latina. Un análisis de cinco experiencias. Santiago de Chile: División de Desarrollo Social de la Comisión Económica para América Latina y el Caribe (CEPAL). 
